\title{
Development of inferences over elementary-school grades: II. Retention of explicit and implicit words
}

\author{
MELVIN H. MARX \\ Florida Institute of Technology, Melbourne, Florida \\ and Western Carolina University, Cullowhee, North Carolina
}

\begin{abstract}
Elementary-school students in Grades 3-8 were read sentences containing either explicit or implicit names of tools. There was a reliable increment over grades in the number of implicit tools recalled, as measured by a word-stem-completion task; a sharp increase occurred from combined third- and fourth-grade classes to combined fifth- and sixth-grade classes, with a lesser increment from the latter to combined seventh- and eighth-grade classes. Smaller, statistically nonreliable increments occurred in recall of explicitly named tools. The results suggest that there is an increment in the generation of inferences over the early grades.
\end{abstract}

Early research reported by Paris, among others, indicated that inferences concerning implicit words increase over the elementary-school grades. For example, Paris and Lindauer (1976) found that first graders were able to recall more study sentences when provided explicit rather than merely implicit cues (e.g., the cue spoon for the sentence, "The truck driver stirred the coffee in his cup with a spoon," compared with the same sentence minus the last three words). Fifth graders, however, were able to recall as many of the study sentences when cued with words that had only been implicit (e.g., spoon when it had not actually been included in the sentence).

Graf (1990) has assembled evidence from a small number of recent studies that suggests that implicit memory, as measured by fragmentary words or pictures, shows little development over the elementary-school years, in contrast with the regular increment typically revealed by explicit-memory tests (usually recall or recognition tests, in which the subject is asked to retrieve studied materials, rather than merely to answer some questions, as the implicit-memory test requires). Moreover, Parkin (1989), using pictorial stimuli, has reported that implicit memory is functional as early as 3 years of age, before effective explicit memory occurs.

These seem to be somewhat contradictory results, if one assumes that the tasks tap the same memory processes. In any event, our understanding of the role of inferences in memory needs a more solid and extensive empirical base than is now available. The present experiment is one of a series of exploratory experiments designed to help

I thank Marvin Oestmann, Principal, and participating teachers at Redeemer Lutheran School, Stuart, FL, for the collection of the data and Bruce Henderson for the analysis of variance. Address communications to M. H. Marx, Department of Psychology, Western Carolina University, Cullowhee, NC 28723. fill in some of the gaps in our knowledge of how inferences, especially as they are generated by implicit words, develop and are retained and used over the elementaryschool grades.

\section{METHOD}

\section{Subjects}

The subjects were 87 students in the third through the eighth grades in a small private school. (The answer sheets of an additional 3 students were discarded because of failure to follow instructions.) Classes in contiguous grades were paired, for control purposes, in the experiment. The resulting $n s$ (with mean ages and $S D$ s) were: Grades 3-4, 27 (9.6 years, .79 year); Grades 5-6, 30 (11.8 years, .86 year); and Grades 7-8, 30 (13.4 years, .58 year).

\section{Experimental Design}

Retention of explicit and implicit words was compared over grades, with the explicit-implicit variable controlled by counterbalanced sets of stories. Set A stories were given to Grades 3, 5, and 7, and Set B stories, with reversal of the explicit-implicit condition for specific tools, to Grades 4, 6, and 8 .

\section{Stimulus Materials}

There were 14 one-sentence "stories." The first and the last were buffers, the same in both sets. The 12 sentences that were scored were randomly ordered, except that no more than 2 successive explicit or implicit sentences were permitted.

The word-stem retention test consisted of 18 three-letter stems, with the remaining letters of each word represented by an appropriate number of blank spaces. There were also two practice items. There were six explicitly named tools, six implicit tools, and six new (unstudied) tools. ${ }^{1}$ The three types of test item were randomly distributed, except that two of each type were included in each third of the test list. The test was administered under explicit, or declarative, conditions; subjects were told that all of the incomplete words were tools and that some of them had been in the sentences read earlier.

The Appendix contains the story sentences, with the tool names, and the word-stem test items.

\section{Procedure}

The data were collected by the teachers in their own intact classes. Each teacher was given detailed procedural instructions. The students were told that they would be read some short sentences and that they should 
try to remember the "stories" in the sentences because questions would be asked about them later. There was a 2 -sec pause between sentences.

After the sentences were read, answer sheets were passed out, and the students were asked to fill in some factual information at the top, such as their birthdates. The teacher explained that the memory test would consist of three-letter word stems, all of which spelled out names of tools when completed, and that some of the tools had been in the sentences read to them and some had not. Two practice items were given: TRA(ctor), from the recency buffer, and CHA(insaw), which was a new word. Approximately $5 \mathrm{~min}$ were allowed for the test.

\section{RESULTS}

The mean number of explicit, implicit, and new words successfully completed on the word-stem test is listed for each grade level in Table 1 . Children's scores were analyzed in a 3 (grade level) $\times 3$ (word type) analysis of variance with word type as a repeated measure. The main effects of grade level $[F(2,86)=16.60]$ and word type $[F(2,172)=11.06]$ were reliable at the .01 level, but were conditioned by a grade level $\times$ word-type interaction $[F(4,172)=4.37, p<.01]$. Simple main effects analyses at each grade level indicated a word-type effect for the combined third- and fourth-grade group $[F(2,54)=$ $14.50, p<.01]$ and for the combined fifth- and sixthgrade group $[F(2,60)=3.65, p<.05]$, but not for the combined seventh-and eighth-grade students $[F(2,58)=$ 1.02]. Comparisons of mean correct word types for each grade level with $t$ tests revealed three reliable differences. Third and fourth graders completed more explicit than implicit words $[t(27)=4.90, p<.01]$ and more explicit than new words $[t(27)=3.73, p<.01]$. Fifth and sixth graders successfully completed more explicit than new words $[t(30)=2.96, p<.01]$. No other word-type comparisons within grades were reliable.

A direct comparison of recall of explicit and implicit words was also made on an individual basis over the three grade levels. The percentage of subjects who successfully completed a higher proportion of explicit as opposed to implicit word stems declined sharply over grade levels. The proportions were $74 \%$ ( 20 of 27 ), $53 \%$ (16 of 30 ), and $43 \%$ (13 of 30) for Grades 3-4, 5-6, and 7-8, respectively. The decline from the lowest to the highest pair of grades was reliable $\left[\chi^{2}(1)=5.59, p<.05\right]$.

\section{DISCUSSION}

The major conclusion supported by these data is that the making of inferences, at least as measured by word-stem completions of implicit words, develops over the elementary-school grades. ${ }^{2}$ The present results are consistent with those reported earlier by Paris and Lindauer (1976): Fifth-

Table 1

Mean Number of Words Completed by Word Type and Grade Level

\begin{tabular}{cccc}
\hline & \multicolumn{3}{c}{ Word Type } \\
\cline { 2 - 4 } Grade Level & Explicit & Implicit & New \\
\hline $3-4$ & 4.1 & 2.6 & 3.0 \\
$5-6$ & 4.4 & 3.8 & 3.6 \\
$7-8$ & 4.5 & 4.3 & 4.7 \\
\hline
\end{tabular}

to eighth-grade students seem to be about as likely to process an implicit word, presumably after inferring it, as they are to process an explicitly named one, whereas this cannot be said of students in the lower grades.

It should be noted that although the word-stem-completion task used in this experiment is one of the major ones used in tests of implicit memory, the instructions that preceded it were of the usual explicit-memory type. Graf and Mandler (1984) have shown that exactly the same study materials and word-stem test items produce quite different results, depending on the type of instructions-implicit (write down the first word that comes to mind) or explicit (remember and write down words from the study list). Thus the apparently contradictory relationship between the present results and Graf's (1990) recent suggestion that implicit memory itself shows little change over elementary-school grades cannot be readily explained on the basis of existing data.

The present data do seem to have some relevance for another persistent theoretical issue, that of whether the temporal locus of inference generation occurs at comprehension or at retrieval. This issue, which has been comprehensively reviewed by Singer (1988), was first raised following Bransford and Franks's (1971) seminal demonstration that implicit words can cue identification of study sentences. Several reports provided evidence that appeared to support the comprehension hypothesis (e.g., Johnson, Bransford, \& Solomon, 1973, as well as Paris \& Lindauer, 1976 , cited earlier). Alternative interpretations were then suggested as a result of subsequent experiments (e.g., Corbett \& Dosher, 1978; Singer, 1979a, 1979b).

In all of the research just mentioned, study-sentence identification was used as the primary dependent variable. Use of the word-stem-completion task in the present experiment makes certain alternative interpretations (Singer, 1988) irrelevant-for example, the argument that presenting an implicit word as a test cue can serve to mediate sentence identification, such as spoon's making the subject think of "stirring," so that the subject is then able to think also of the study sentence, "The truck driver stirred the coffee in his cup." Critical evaluation of the present data will require a different set of theoretical filters. Taken at face value, however, the results do seem to offer support for the proposition that inferences are made at comprehension, at least under some conditions, and are subsequently effective in the completion of word stems. In any event, the use of the inferential words themselves, rather than the study sentences, offers a simpler, more direct kind of test and one that seems to be less readily open to alternative interpretations.

\section{REFERENCES}

Bransford, J. D., \& Franks, J. J. (1971). The abstraction of linguistic ideas. Cognitive Psychology, 2, 231-250.

Corbett, A. T., \& Dosher, B. A. (1978). Instrument inferences in sentence encoding. Journal of Verbal Learning \& Verbal Behavior, 17, 479-492.

GrAF, P. (1990). Life-span changes in implicit and explicit memory. Bulletin of the Psychonomic Society, 28, 353-358.

GrAF, P., \& MANDLER, G. (1984). Activation makes words more accessible but not necessarily more retrievable. Journal of Verbal Learning \& Verbal Behavior, 23, 553-568.

Johnson, M. K., BRANSFord, J. D., \& Solomon, S. K. (1973). Memory for tacit implications of sentences. Journal of Experimental Psychology, 98, 203-205.

Paris, S., \& Lindauer, B. K. (1976). The role of inference in children's comprehension and memory for sentences. Cognitive Psychology, 8, 217-227.

PARKIN, A. J. (1989). The development and nature of implicit memory. In S. Lewandowsky, J. Dunn, \& K. Kirsner (Eds.), Implicit memory: Theoretical issues (pp. 231-240). Hillsdale, NJ: Erlbaum.

Singer, M. (1979a). Processes of inference during sentence encoding. Memory \& Cognition, 7, 192-200.

SiNGER, M. (1979b). Temporal locus of inferences in the comprehension of brief passages: Recognizing and verifying inferences about instruments. Perceptual \& Motor Skills, 49, 539-550.

SINGER, M. (1988). Inferences in reading comprehension. In M. Daneman, G. MacKinnon, \& T. Waller (Eds.), Reading research: Advances in theory and practice (Vol. 7, pp.177-219). New York: Academic Press. 


\section{NOTES}

1. Set B sentences, however, had only 5 sentences with explicit tools; because of a typing error, the word razor was not used explicitly. Appropriate prorated adjustments were made in the scores for Grades 4, 6, and 8.
2. The fact that one sentence, containing an explicit tool, was omitted from Set B applied equally to the three pairs of grades. Moreover, it was unrelated to the implicit-memory measure, which was the primary concern of the experiment. The relatively small but steady increments in recall of explicitly presented words are typical of the results from many studies, the failure of the measure to reach statistical reliability notwithstanding.

APPENDIX

Study and Test Materials

Set A Sentences
The shopper signed a check to pay for the
TV set.
TV set.

The fisherman sliced the large fish into equal sections [with his knife].

A boy pounded a nail into the side of a wooden box.

A teacher put the next assignment on the blackboard.

The homeowner [bought a drill and] bored some holes in the new door.

A woman darned her son's socks [with a needle].

The young lady tried to shield her head from the sudden rain and just missed poking another pedestrian in the eye.

The barber gave the young man a very close shave.

A preschooler colored in half of the pictures in his book [with a crayon].

The housewife swept the kitchen floor clean.

A student [took an eraser and] wiped all the figures off the blackboard.

A mother fed cereal from a small dish to her baby.

The workman [got a shovel and] dug a large hole for the tree.

The farmer went to the bank before buying a new tractor.

Set B Additions $\quad$ Word Stems

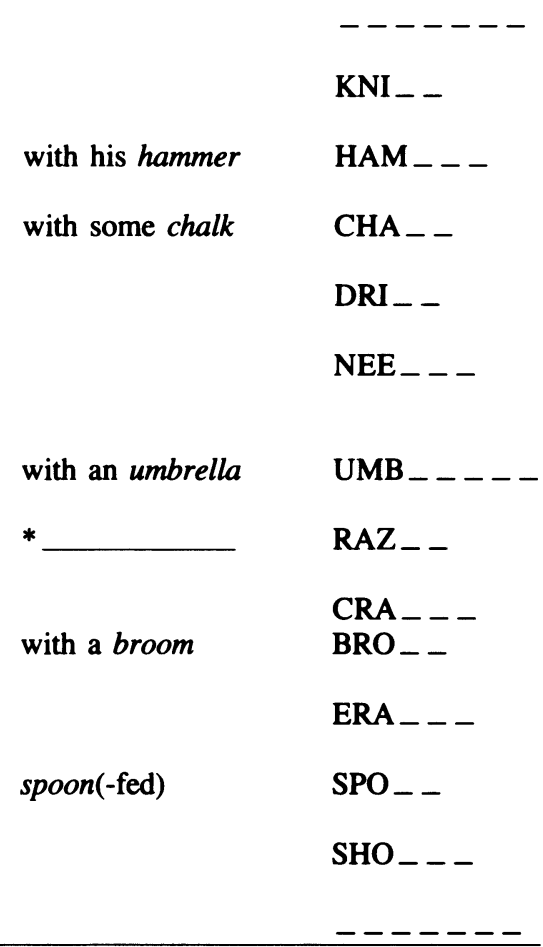

Note-Key words are italicized; bracketed phrases in Set A sentences were omitted from Set B sentences. New word stems (with completions) were: SCI(ssors), PEN(cil), PLI(ers), SWO(rd), PAD(dle), RIF(le). $\quad$ *This sentence was inadvertently omitted from Set B so that the word razor was not used explicitly. 